
\title{
25 Research Square \\ Inferior Vena Cava Filter Thrombosis Associated With a Free-Floating Thrombus on a Retrieval Hook: A Case Report and Literature Review
}

\section{Ruihua Duan}

Guangzhou University of Traditional Chinese Medicine First Affiliated Hospital

\section{Weihao Li}

Guangzhou University of Traditional Chinese Medicine First Affiliated Hospital

Min Liu

Guangzhou University of Traditional Chinese Medicine First Affiliated Hospital

Chenhui Bai

Guangzhou University of Traditional Chinese Medicine First Affiliated Hospital

Yisheng Lin ( $\nabla 104220421 @ q q . c o m$ )

Guangzhou University of Traditional Chinese Medicine First Affiliated Hospital

\section{Fangjun Wang}

Guangzhou University of Traditional Chinese Medicine First Affiliated Hospital

\section{Case report}

Keywords: inferior vena cava filter thrombosis, inferior vena cava filter, deep vein thrombosis, endovascular procedures

Posted Date: July 7th, 2021

DOl: https://doi.org/10.21203/rs.3.rs-669740/v1

License: (c) (i) This work is licensed under a Creative Commons Attribution 4.0 International License. Read Full License 


\section{Abstract}

Background: It has been widely reported that IVCFT is a type of thrombotic disease. However, there are very few case reports regarding free-floating thrombi on retrieval hooks.

Case presentation: This case report describes inferior vena cava filter thrombosis (IVCFT) associated with a free-floating thrombus on a retrieval hook in a 26-year-old male. Rotational digital subtraction angiography (RDSA) was used for the diagnosis and a second retrievable filter was placed above the original filter to prevent detachment of the thrombi. Catheter-directed thrombolysis (CDT) and aspiration thrombectomy (AT) were used to treat the thrombosis, and both filter retrievals were performed successfully.

Conclusions: Attention should be paid to the diagnosis and endovascular procedures of a free-floating thrombus on a retrieval hook. In the event of thrombosis, the therapeutic effect and safety level can be increased through CDT combined with AT.

\section{Background}

Inferior vena cava (IVC) filters can effectively reduce the risks of venous thromboembolism (VTE) and the morbidity and mortality of pulmonary embolism (PE) ${ }^{[1]}$. However, the detachment of free-floating thrombi and primary thrombosis caused by the filter may lead to inferior vena cava filter thrombosis (IVCFT) ${ }^{[2]}$, which could result in IVC obstruction or increase the incidence of $\mathrm{PE}^{[3]}$. To date, no report has been found on IVCFT associated with a free-floating thrombus on a retrieval hook. Hence, we present a case of the diagnosis and interventional therapy of IVCFT associated with a free-floating thrombus on a retrieval hook with a literature review.

\section{Case Report}

A 26-year-old male underwent skin grafting to the right calf due to sharp instrument injury. Postsurgical swelling aggravated his right lower limb. On the fourth day after surgery, ultrasonography of the lower extremity revealed right iliac vein and femoral vein thrombosis (complete) and a D-dimer of $9.23 \mathrm{mg} / \mathrm{L}$. The patient was diagnosed with mixed acute deep vein thrombosis of the right lower extremity. Catheterdirected thrombolysis (CDT) was performed after Günther Tulip retrievable filter (Cook Medical Inc., Bloomington, IN, USA) placement (Fig. 1). Postoperative systemic anticoagulant therapy was then performed. After surgery, swelling in the right lower limb was obviously alleviated and the patient was discharged at the fifth day after thrombolysis. Regular oral rivaroxaban was administered after discharge.

The patient returned to the hospital 45 days after filter placement and was scheduled to undergo IVC filter retrieval. No swelling was observed in the patient's right lower limb. Doppler ultrasonography of the lower limb indicated that the right lower extremity deep veins were unobstructed with no thrombosis and a Ddimer of $0.73 \mathrm{mg} / \mathrm{L}$. 
Posteroanterior (PA) views of the lower IVC via right jugular vein puncture suggested unobstructed blood flow and no filter migration. A sub-round filling defect was detected in the filter and a round filling defect was noted floating above the filter retrieval hook. A pigtail catheter was introduced below the filter through a sheath. Rotational digital subtraction angiography (RDSA) and 3D reconstruction were then performed indicating that a filling defect was seen in the retrieval hook and IVC filter, but no filter tilt was detected. A retrieval Günther Tulip filter was implanted above the original filter. Urokinase $(400,000 \mathrm{IU} / \mathrm{d})$ was then injected through the pigtail catheter for thrombolysis therapy (Fig. 1).

IVC angiography after 5 days suggested no obvious change in the filling defect in the original filter or the retrieval hook and no obvious filling defect in the retrievable filter placed above. A short vascular $9 \mathrm{~F}$ sheath was implanted via right femoral superficial vein puncture, while a long $7 \mathrm{~F}$ sheath $(90 \mathrm{~cm})$ was introduced to the original filter. Multiple red and pale thrombi were then removed through negative pressure aspiration thrombectomy (AT) (Fig. 2). Another angiography showed reduced filling defects in the original filter and no obvious filling defect in the second filter. The original filter was successfully retrieved via the right jugular vein. The angiography of the sheath via right femoral vein demonstrated multiple filling defects (Fig. 1). The long 7F sheath $(90 \mathrm{~cm}$ ) was then inserted into the second retrievable filter to remove red and pale thrombi (mainly pale) through negative pressure AT. The angiography was conducted again, resulting in obviously reduced filling defects in the second filter, which was then successfully retrieved via the right jugular vein. Another angiography showed that the IVC was unobstructed with no stenosis or extravasation of contrast agents. Angiography of the main pulmonary artery via pigtail catheter insertion indicated no obvious filling defects in the main pulmonary artery and the primary to tertiary bronchi. Sparse blood vessels in the middle-upper part of the right peripheral lung suggested the possibility of small PE.

The patient did not develop symptoms such as cough, hemoptysis, chest congestion, chest pain, or dyspnea. ECG monitoring indicated a normal blood oxygen saturation level. The patient was discharged on day 2 after the procedure. A 6-month follow-up showed that the patient's symptoms completely resolved and he was able to bear weight and walk comfortably.

\section{Discussion}

According to research ${ }^{[4]}$, the incidence of IVCFT in 598 cases of filter implantation was approximately $18.6 \%$, of which 4 cases were associated with thrombosis above the filter. A prior study ${ }^{[5]}$ reported 1 case of IVC filter thrombosis on a retrieval hook of a filter tilted and attached to the IVC wall, in which the thrombus detached without endovascular therapy. However, no report has been found on IVCFT associated with a free-floating thrombus on retrieval hook endovascular therapy.

Causes of free-floating thrombi on retrieval hooks

The causes of IVCFT mainly include the following: (1) free-floating thrombi are captured, (2) the filter stimulates the IVC wall resulting in IVCFT, and (3) iliac vein thrombi extend into the filter and lead to 
$\mathrm{IVCF}^{[6]}$. In addition, factors including various shapes of filters produced by different manufacturers, filter implantation time, blood hypercoagulability (cancer, obesity, pregnancy, antiphospholipid syndrome, etc.), and patient age can cause IVCFT ${ }^{[7]}$. In our case, no tilt was observed in the IVC filter and standard anticoagulant therapy was initiated. The filling defects in the filter are considered to be detached thrombi captured by the filter. When there is a small amount of thrombus in the filter, if the IVCF is close to the renal vein, the fluid dynamics of the renal venous vein will be affected. High wall shear stress (WSS) occurs along the caval wall downstream from the thrombus in infrarenal filter positions. The lower the WSS, the slower the blood flow velocity in the section, leading to an increased possibility of thrombosis. When the IVCF is placed just below or near the level of the renal vein, the lowest WSS is close to the low flow area downstream of the bilateral renal veins ${ }^{[8]}$. This is the recovery hook location (Fig. 3). Therefore, the causes of a free-floating thrombus on a retrieval hook could be as follows: a vortex of venous return forms near the retrieval hook after the detached IVC thrombi are captured by the filter, leading to platelet adhesion, so stagnation and even coagulation can occur among platelets. Blood cells are wrapped in fibrin strands and eventually form mixed thrombi (Fig. 2).

Diagnosis of free-floating thrombi on retrieval hooks

Most IVCFT patients have no clinical symptoms ${ }^{[9]}$. Generally, IVCFT is diagnosed when the patient is scheduled to undergo filter retrieval surgery and DSA detects filling defects in the filter. This examination is the gold standard for IVCFT diagnosis. Zhang et al. ${ }^{[10]}$ conducted multi-angle IVC angiography on 1 patient with suspected IVCFT in which the filling defects were eventually assessed as artifacts in the filter; hence, artifacts in filters should not be overlooked. RDSA conducts data acquisition and imaging on selected areas using a fast rotating $\mathrm{C}$-arm, acquires dynamic subtraction images from different continuous angles, and generates $\mathrm{CT}$-like images through 3D reconstruction to provide more accurate and detailed image information ${ }^{[11]}$. IVC angiography using RDSA can avoid the interference of intestinal gas and centrum while improving the accuracy of IVCFT diagnosis. Moreover, RDSA can initiate multi-angle and dynamic observation on IVC thrombi. The round filling defect floating above the retrieval hook in our case had a candlelight shape, which was unattainable via regular IVC enhanced CT scan.

Endovascular treatment of free-floating thrombi on retrieval hooks

IVCFT therapies involve anticoagulants, CDT, and PMT. The American College of Chest Physicians (ACCP) in Antithrombotic Therapy for VTE Disease: CHEST Guidelines ${ }^{[12]}$ recommend that when there are no anticoagulant medication contraindications, anticoagulant therapy should be initiated after filter placement. New oral anticoagulants with low risks of bleeding and no need for INR measurement such as rivaroxaban are also recommended. A prior study ${ }^{[7]}$ indicated that once IVCFT is observed, the patient should undergo immediate anticoagulant therapy as soon as anticoagulant medication contraindications are ruled out, and the possibility of fatal PE and serious complications of the lower extremity should be assessed. In cases of low possibilities, anticoagulant therapy should continue and angiography reexamination should be performed in 2 to 3 weeks; in cases of high possibilities, further treatment is required. 
CDT ensures the effect of thrombolysis while reducing the dosage and thrombolysis time, significantly lowering the risk of bleeding and benefitting patients the most ${ }^{[12]}$. CDT is superior to peripheral venous thrombolysis (PVT) ${ }^{[13]}$. Aiming at IVCFT, Tian et al. ${ }^{[14]}$ noted that the therapeutic effect of local CDT far exceeded that of PVT and the filter removal rate in a local CDT group was higher than that in a PVT group.

In our case, the therapeutic effect of oral rivaroxaban combined with standard anticoagulant therapy and CDT for 5 days was not satisfactory, which could have been caused by the following: (1) in CDT therapy, the catheter's side hole of the is usually placed inside the thrombi; however, it is relatively difficult to insert the wire through retrieval hook thrombi, which is especially true for free-floating thrombi; hence, thrombolytic drugs have only limited access to the thrombi; (2) thrombolytic drugs in the IVC filter may be removed by venous return before the thrombi is fully accessed; (3) the thrombi formation time is rather long, while the therapeutic effect of CDT depends on the age and thrombi load. The thrombolytic effect of chronic thrombosis is not ideal; therefore, the combination of AT or percutaneous mechanical thrombectomy (PMT) with CDT could be more effective for removing thrombi and reducing the dosage of thrombolytic drugs and the thrombolysis time, which is superior to the exclusive use of either therapy ${ }^{[15,16]}$. Free-floating thrombi on retrieval hooks have a detachment risk; hence, new retrievable filters should be placed above original filters to prevent secondary PE caused by retrieval hook thrombi detachment during AT. There are also indications ${ }^{[17]}$ that when the IVCFT load is relatively heavy and the thrombi are larger than $50 \%$ of the IVC diameter, retrieval filters should be placed before AT. For AT therapy against IVCFT, aspiration catheters should be placed as close as possible to the thrombi to reduce the amount of bleeding, since the blood flow around the thrombi is relatively heavy; hence, adjustable curved sheaths or MPA catheters with a diameter over $8 \mathrm{~F}$ are suggested. RDSA excels at locating the positions of thrombi and guiding CDT catheters. Retrieval hook thrombi, free-floating thrombi in particular, should be crushed by the catheter and captured by the filter above after detachment and then removed by AT. Filters should be retrieved as soon as possible after IVCFT removal to eliminate further risks.

When IVCFT develops into chronic thrombosis, CDT and PMT may no longer effectively remove the thrombi, so balloon dilatation and stent implantation should be considered to reconstruct the IVC. Branco et al. ${ }^{[18]}$ initiated balloon dilatation on 4 patients and stent implantation on 2 out of 9 IVCFT patients, and the occlusions were successfully removed. Cui et al. ${ }^{[19]}$ implanted IVC stents in 40 IVCFT patients. The stents and filters were in good shape with no serious bleeding or $\mathrm{PE}$, and the restenosis rate was $7.7 \% 12$ months after surgery. Marmagkiolis et al. ${ }^{[20]}$ reported successful IVC reconstruction with PMT, balloon dilatation, and stent implantation on 1 IVCFT patient. Self-expandable braided stents are recommended (such as Wallstent) ${ }^{[21]}$. The stents can be placed either parallel or through the filter in accordance with the filter shape; that is, the filter can be regarded as the reference for stent anchorage. In brief, it is both feasible and safe to implant stents in patients with chronic IVCFT to remove occlusions, whereas the long-term patency rate of stents still needs to be further improved ${ }^{[20-23]}$. 


\section{Conclusion}

A vortex of venous return formed near the retrieval hook caused by thrombi in the filter leading to a freefloating thrombus on non-tilting IVC filter retrieval hook thrombosis. RDSA can provide a reliable diagnosis of IVCFT associated with retrieval hook thrombosis. Retrievable filter implantation above the original filter is an effective method of preventing symptomatic PE caused by thrombi detachment. If thrombosis occurs, the therapeutic effect and safety level can be improved by CDT combined with AT.

\section{Abbreviations}

IVC: inferior vena cava

IVCFT: inferior vena cava filter thrombosis

VTE: venous thromboembolism

PE: pulmonary embolism

CDT: catheter-directed thrombolysis

AT: aspiration thrombectomy

RDSA: rotational digital subtraction angiography

PVT: peripheral venous thrombolysis

PMT: percutaneous mechanical thrombectomy

\section{Declarations}

-Ethical Approval and Consent to participate: The study is approved by the Ethics Committee of the first affiliated hospital of Guangzhou university of TCM. The patient has given consent to participate in the research and to have the case published in an academic journal.

-Consent for publication: Written informed consent for publication was obtained from all participants.

-Availability of supporting data: This manuscript is original and has not been published and will not be submitted elsewhere for publication while being considered by Thrombosis Journal. And the study is not split up into several parts to increase the quantity of submissions and submitted to various journals or to one journal over time. No data have been fabricated or manipulated (including images) to support our conclusion. No data, text, or theories by others are presented as if they were our own.

-Competing interests: The Authors declare that there is no conflict of interest. 
-Funding: 1. Experimental study of targeted allicin nanomicrospheres in the treatment of implanted hepatocellular carcinoma in rats by transarterial perfusion $₫ 2015 a 020211024$, Department of Science and Technology of Guangdong Province

2. Experimental study on the treatment of hepatocellular carcinoma by transarterial perfusion of thermalsensitive microgel loaded with CRGD and cantharidin $₫ 2019 A 1515012118 \otimes T h e$ Natural Science Foundation of Guangdong Province, China

-Authors' contributions: authors whose names appear on the submission have contributed sufficiently to the scientific work and therefore share collective responsibility and accountability for the results.

-Acknowledgments: I express my gratitude to Mrs. Mei Huang for her suggestions. We thank all of the nurses on the interventional radiology staff (in particular Mrs. Qiong Liu) for their continued assistance.

-Ethics statement: This manuscript is original and has not been published and will not be submitted elsewhere for publication while being considered by Thrombosis Journal. And the study is not split up into several parts to increase the quantity of submissions and submitted to various journals or to one journal over time. No data have been fabricated or manipulated (including images) to support our conclusion. No data, text, or theories by others are presented as if they were our own.

The submission has been received explicitly from all co-authors. And authors whose names appear on the submission have contributed sufficiently to the scientific work and therefore share collective responsibility and accountability for the results.

-Conflict of interest: The Authors declare that there is no conflict of interest.

\section{References}

1. Farge $D$, Debourdeau $P$, Beckers $M$, et al. International clinical practice guidelines for the treatment and prophylaxis of venous thromboembolism in patients with cancer. J Thromb Haemost, 2013, 11(1): 56-70.

2. Joels C S, Sing R F, Heniford B T. Complications of inferior vena cava filters. Am Surg, 2003, 69(8): 654-9.

3. Golowa Y, Warhit M, Matsunaga F, et al. Catheter directed interventions for inferior vena cava thrombosis. Cardiovasc Diagn Ther, 2016, 6(6): 612-622.

4. Ahmad I, Yeddula K, Wicky S, et al. Clinical sequelae of thrombus in an inferior vena cava filter. Cardiovasc Intervent Radiol, 2010, 33(2): 285-9.

5. Momose T, Shimizu T, Misawa T, et al. A Case of Inferior Vena Cava Filter Thrombus on a Retrieval Hook of the Filter[M]. 56. 2016: 55-58.

6. Avgerinos E D, El-Shazly O, Jeyabalan G, et al. Impact of inferior vena cava thrombus extension on thrombolysis for acute iliofemoral thrombosis. J Vasc Surg Venous Lymphat Disord, 2016, 4(4): 385- 
91.

7. Habito C R, Kalva S P. Inferior vena cava filter thrombosis: a review of current concepts, evidence, and approach to management. Hosp Pract (1995), 2011, 39(3): 79-86.

8. Wang S L, Singer M A. Toward an optimal position for inferior vena cava filters: computational modeling of the impact of renal vein inflow with Celect and TrapEase filters(1535-7732 (Electronic)).

9. Nazzal M, Chan E, Nazzal M, et al. Complications related to inferior vena cava filters: a single-center experience. Ann Vasc Surg, 2010, 24(4): 480-6.

10. Qian S, Zhang C. Prevention and Treatment of inferior vena cava filter thrombosis. Chinese Journal of Vascular Surgery(Electronic Version), 2013, 5(3): 153-154+157.(in Chinese)

11. Gailloud P, Oishi S, Carpenter J, et al. Three-dimensional digital angiography: new tool for simultaneous three-dimensional rendering of vascular and osseous information during rotational angiography. AJNR Am J Neuroradiol, 2004, 25(4): 571-3.

12. Kearon C, Akl E A, Ornelas J, et al. Antithrombotic Therapy for VTE Disease: CHEST Guideline and Expert Panel Report. Chest, 2016, 149(2): 315-352.

13. Alesh I, Kayali F, Stein P D. Catheter-directed thrombolysis (intrathrombus injection) in treatment of deep venous thrombosis: a systematic review. Catheter Cardiovasc Interv, 2007, 70(1): 143-8.

14. Tian X, Wang S, Liu J L, et al. A prospective clinical study on the efficacy and safety of catheterdirected thrombolysis compared with systemic thrombolysis in Deep Venous Thrombosis subjects with stent . Journal of Capital Medical University, 2015, 36(1): 53-57. (in Chinese)

15. Li W D, Li C L, Qian A M, et al. Catheter-directed thrombolysis combined with manual aspiration thrombectomy for acute inferior vena cava filter thrombosis. Int Angiol, 2016, 35(6): 605-612.

16. Lin P H, Zhou W, Dardik A, et al. Catheter-direct thrombolysis versus pharmacomechanical thrombectomy for treatment of symptomatic lower extremity deep venous thrombosis. Am J Surg, 2006, 192(6): 782-8.

17. Ai W J, Lin S M, Xiao J B, et al. Analysis of therapeutic effect of endovascular treatment for 18 cases of inferior vena cava filter thrombosis. Chinese Journal of Vascular Surgery(Electronic Version), 2018, 10(04): 279-282.(in Chinese)

18. Branco B C, Montero-Baker M F, Espinoza E, et al. Endovascular management of inferior vena cava filter thrombotic occlusion. Vascular, 2018, 26(5): 483-489.

19. Cui C Y, Yin M Y, Huang X T, et al. Clinical analysis of endovascular treatment of filter-related inferior vena cava thrombosis. Chinese Journal of Vascular Surgery(Electronic Version), 2018, 10(01): 1619+23.(in Chinese)

20. Marmagkiolis K, Lendel V, Cilingiroglu M. Endovascular management of IVC syndrome after IVC filter placement. Rev Port Cardiol, 2015, 34(9): 555.e1-4.

21. Partovi S, Kalva S P, Walker T G, et al. Long term follow-up of endo-vascular recanalization of chronic inferior vena cava occlusion secondary to inferior vena cava filters. Vasa, 2017, 46(2): 121-126. 
22. Banerjee S, Patel H M, Sheorain V K, et al. Double Barrel In Situ Recanalization of Thrombosed Nonretrievable IVC filter. Ann Vasc Surg, 2016, 33: 229.e11-5.

23. Arabi M, Krishnamurthy V, Cwikiel W, et al. Endovascular treatment of thrombosed inferior vena cava filters: Techniques and short-term outcomes. Indian J Radiol Imaging, 2015, 25(3): 233-8.

\section{Figures}
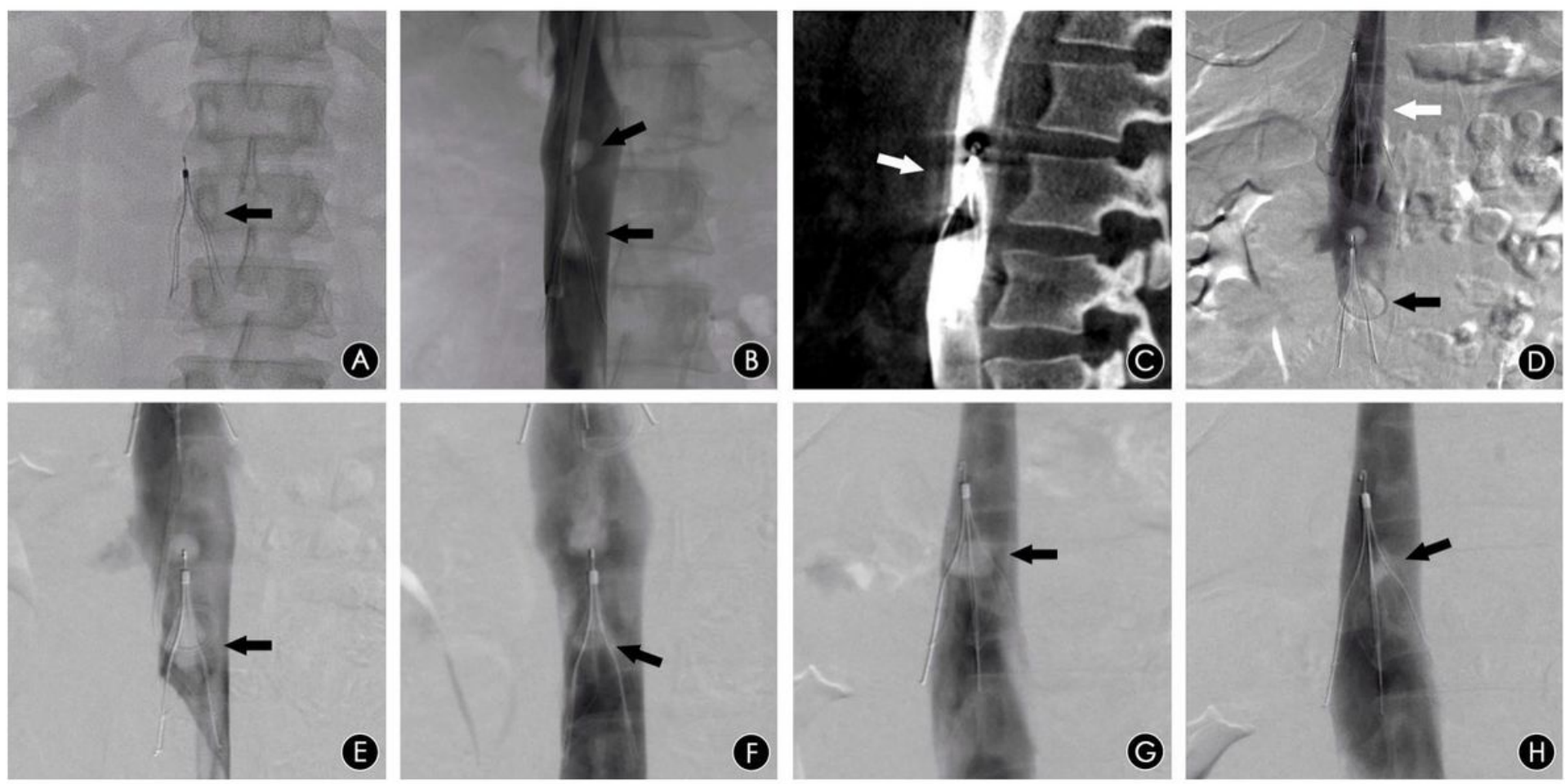

\section{Figure 1}

(A) The filter was placed at the level of the superior border of the L2 vertebra. (B) IVC angiography indicated filling defects in the filter. The round filling defect floating above the retrieval hook appeared to have a candlelight shape. (C) RDSA and 3D reconstruction revealed IVC filter and retrieval hook thrombosis. (D) A second retrievable filter was placed above the original filter (white arrow); the tip of a pigtail catheter was placed in the original filter for thrombolysis (black arrow). (E) IVC angiography suggested that IVCFT had no obvious change. (F) The thrombi in the original filter were partially removed after AT. (G) The original filter was retrieved, and the detached retrieval hook thrombi were captured by the second filter's umbrella-shaped section (black arrow). $(\mathrm{H})$ The thrombi in the second filter were mostly removed after AT. 

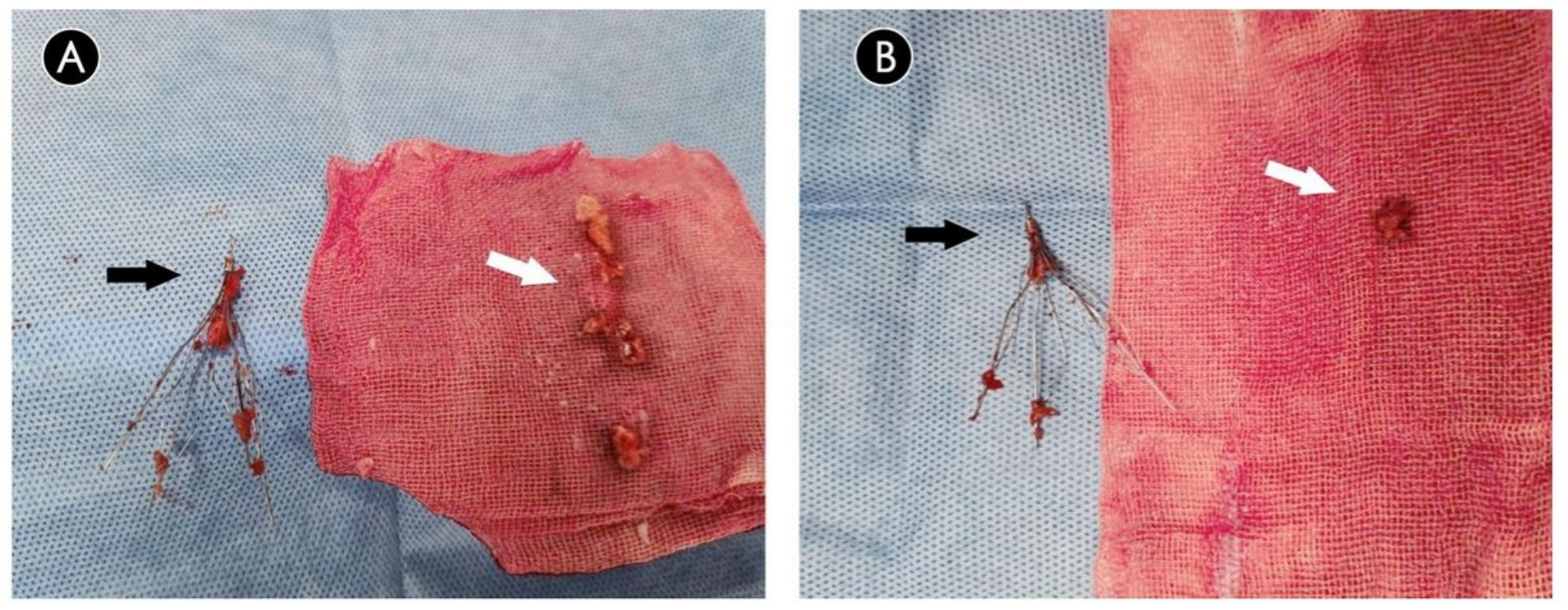

\section{Figure 2}

(A) A small number of thrombi were attached to the umbrella-shaped section of the original retrieved filter (black arrow). Several red and pale thrombi were removed after AT, some the color of salmon (white arrow). (B) The shape of the second retrieved filter (black arrow) and the red and mostly pale thrombi after AT (white arrow).
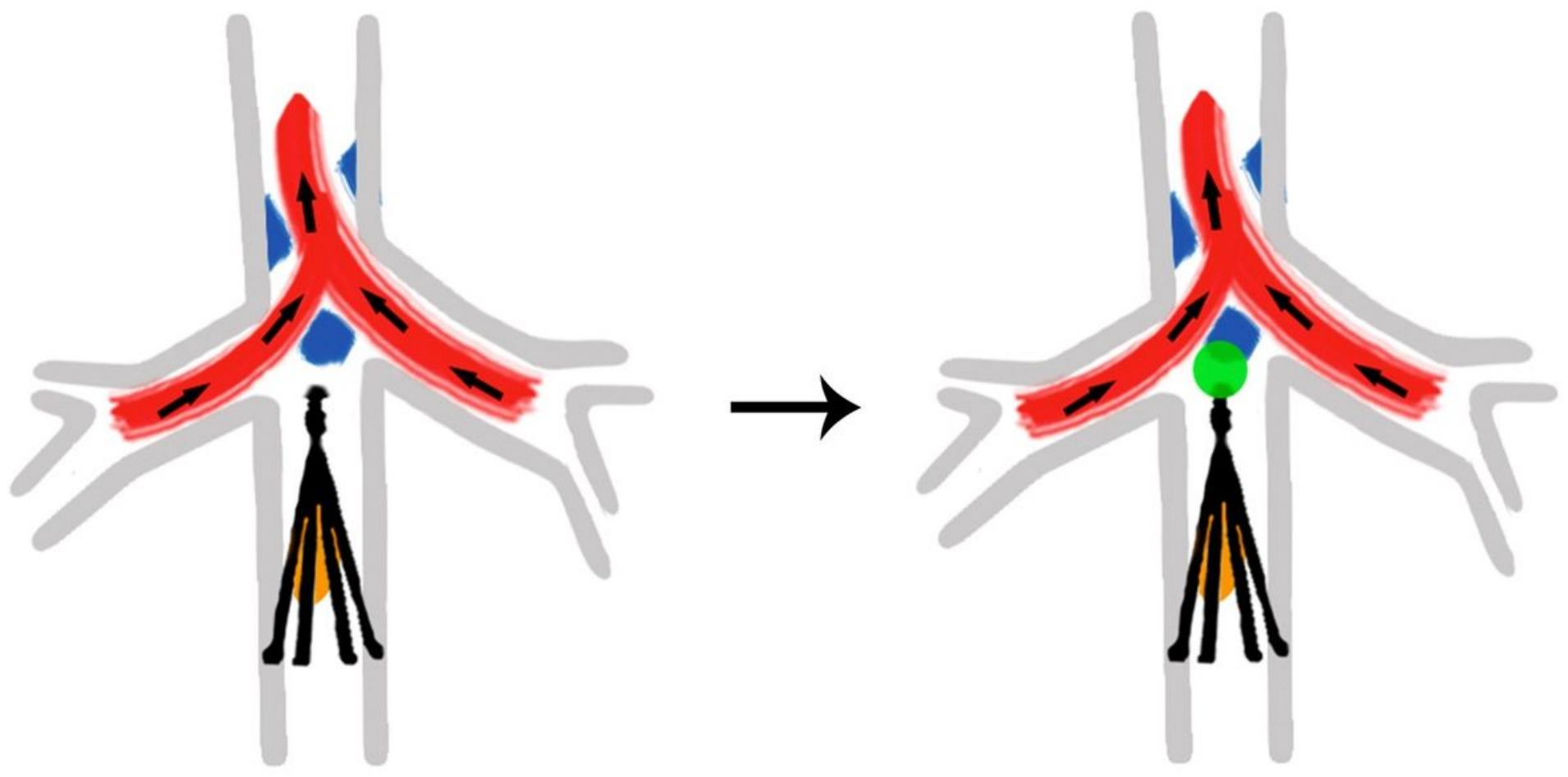

\section{Figure 3}

Fluid dynamics simulation of blood flow in IVC IVCFT (orange); high WSS area (red); low WSS area (blue); retrieval hook thrombosis (green). 